\title{
The Influence of Antibiotics Usage on Extended-spectrum $\beta$-lactamase-producing Enterobacter Colonization among Intensive Care Unit Patients
}

\author{
Wayan Suranadi ${ }^{1}$, Dwi Fatmawati ${ }^{2}$, Christopher Ryalino ${ }^{1 *}$, I Gusti Agung Gede Utara Hartawan ${ }^{1}$, Ferdi Yanto ${ }^{1}$ \\ ${ }^{1}$ Department of Anesthesiology and Intensive Care, Faculty of Medicine, Udayana University, Bali, Indonesia; ${ }^{2}$ Department of \\ Clinical Microbiology, Faculty of Medicine, Udayana University, Bali, Indonesia
}

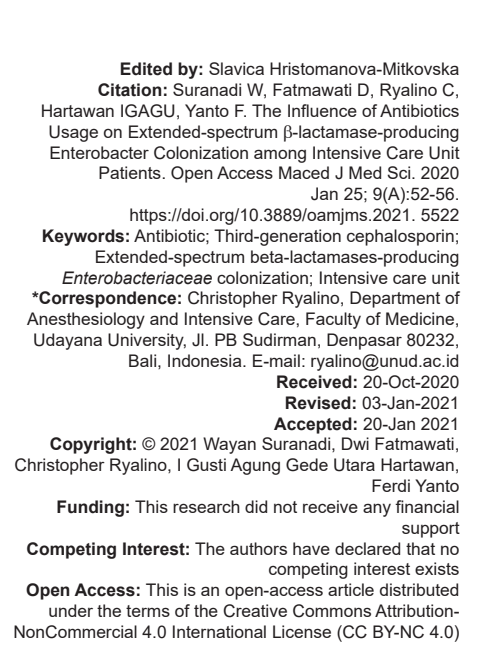

\section{Introduction}

Extended-spectrum $\beta$-lactamases (ESBLs) are bacterial enzymes which are produced to confer resistance to broad range of extended-spectrum $\beta$-lactam antibiotics. The ESBLs hydrolyze extended-spectrum cephalosporins. First reports of ESBLs were in the mid1980 s and mostly Klebsiella pneumoniae and Escherichia coli [1]. In 2013, the Centers for Disease Control and Prevention reported an increasing resistance which included 26,000 ESBL-producing Enterobacteriaceae infections and 1700 deaths in the United States [2], [3].

Colonization of intensive care unit (ICU) patients with ESBL-producing Enterobacteriaceae on admission has an impact on poorer outcome and increasing mortality. A study in Egypt showed that 33\% of the patients admitted to ICU were colonized with ESBL on one or more swab sites. The prevalence of ESBLproducing Enterobacteriaceae found in ICU patients rectal swabs varies throughout the world, $2.25 \%$ in the United States, $15 \%$ in France, $28.2 \%$ in South Korea, and $65 \%$ in India out of which $56 \%$ were ESBL-producing E. coli and 43\% Klebsiella spp. [1], [4], [5].

Risk factors for infection with ESBL-producing organisms are prolonged antibiotic usage, prolonged treatment at ICU, recent invasive procedures, pressure ulcer, anemia, and permanent urinary catheter. Effective and rational usage of antibiotics in ICUs is important for the prevention of the development of antibiotic resistance [5], [6], [7]. The goal of this study was to identify the variables that influence the colonization of Enterobacteriaceae in patients treated at ICU. For the purpose of this study, Enterobacteriaceae are limited to K. pneumoniae and E. coli.

\section{Patients and Methods}

We conducted a prospective cohort study which was approved by the Ethical Committee of Sanglah General Hospital from October 1, 2018, until 
March 31, 2019. Rectal swabs were collected from 70 randomized, adult patients who fulfilled the inclusion and exclusion criteria and willing to sign the informed consent when patients were admitted and discharged from ICU. Inclusion criteria included newly admitted ICU patients aged $>18$ years old who agreed to follow the study protocol after receiving consent to be included in this study. Exclusion criteria included those with known history of allergy to certain antibiotics and those who were treated at the ICU for $<48 \mathrm{~h}$.

Rectal swab specimens were put in transport medium and delivered to the Department of Clinical Microbiology of Sanglah General Hospital to be inoculated in MacConkey medium. After being incubated in $5 \% \mathrm{CO}_{2}$ for $18-24 \mathrm{~h}$, the species were identified and susceptibility was tested using Vitek 2 Compact (BioMerieux, France).

We collected data regarding the study subject's previous antibiotics exposures (type and duration), coexisting conditions, invasive procedures, and other hospitalization-related and demographic information. Categorical variables were presented in percentage while numeric variables were presented in mean \pm deviation standard (SD).

Initial bivariate analysis was conducted using $\chi^{2}$ (Pearson's Chi-square) and considered significant if $p<0.05$. Adjusted relative risk ratio $(R R)$ was used to estimate the influence of the variables to ESBL colonization occurrence rate on the patient who was admitted or discharged from ICU. Statistical analysis was performed using SPSS software (IBM Corp. Released 2013. IBM SPSS Statistics for Windows, Version 22.0. Armonk, NY: IBM Corp.).

\section{Results}

Table 1 shows the general characteristics of the patients. The mean age of the patients was 45.7 \pm 17.75 years old, and $82.8 \%$ were adult (18-64 years old), with length of ICU stay for $6.35 \pm 4.01$ days. There were $57.1 \%$ of male patients with $61.4 \%$ of total patients were from Sanglah General Hospital inward patients. Most patients were exposed to ceftriaxone on admission to ICU. ESBL colonization increased during ICU stay from $25.7 \%$ at admission to $50 \%$ when patients were discharged from ICU (Table 2). The mortality rate was $22.9 \%$ (16 patients).

Our study shows that ICU stay causes an increase on ESBL-producing Enterobacteriaceae colonization rate from $25.7 \%$ (18 patients) at admission to $50 \%$ (35 patients) on ICU discharge with $p=0.003$ ( $p$ $<0.05$ ) and relative risk of 1.994 , with a $95 \%$ confidence interval of 1.225-3.086 (Table 3). The study also finds that respiratory system dysfunction $(p=0.012$,
Table 1: General characteristic

\begin{tabular}{ll}
\hline Variable & $\mathrm{n}=70$ \\
\hline Age (years), mean $\pm \mathrm{SD}$ & $45.7 \pm 17.75$ \\
$18-64$ years old, $\mathrm{n}(\%)$ & $58(82.8)$ \\
$\geq 65$ years old, $\mathrm{n}(\%)$ & $12(17.2)$ \\
Length of stay (days), mean $\pm \mathrm{SD}$ & $6.35 \pm 4.01$ \\
Sex & \\
$\quad$ Male, $\mathrm{n}(\%)$ & $40(57.1)$ \\
$\quad$ Female, $\mathrm{n}(\%)$ & $30(42.9)$ \\
Previously treated at & \\
$\quad$ Other hospital, $\mathrm{n}(\%)$ & $27(38.6)$ \\
$\quad$ Ward, $\mathrm{n}(\%)$ & $43(61.4)$ \\
\hline
\end{tabular}

$\mathrm{RR}=2.828$ ) and antibiotic prescription before ICU admission ( $p<0.001)$ influence ESBL-producing Enterobacteriaceae colonization on patient who was admitted to ICU (Table 4).

Table 2: Patient characteristic when admitted to and discharge from ICU

\begin{tabular}{lll}
\hline Variable & \multicolumn{2}{l}{ Time } \\
\cline { 2 - 3 } & Admitted to ICU & Discharge from ICU \\
\hline Antibiotics & & \\
$\quad$ Ceftriaxone, $\mathrm{n}(\%)$ & $42(60)$ & $38(54.3)$ \\
$\quad$ Cefoperazone, $\mathrm{n}(\%)$ & $10(14.3)$ & $17(24.3)$ \\
$\quad$ Cefazolin, $\mathrm{n}(\%)$ & $13(18.6)$ & $12(17.1)$ \\
$\quad$ Non-cephalosporin, $\mathrm{n}(\%)$ & $5(7.1)$ & $3(4.3)$ \\
Antibiotic usage duration & & \\
$\quad<3$ days, $\mathrm{n}(\%)$ & $51(72.9)$ & $21(30)$ \\
$\geq 3$ days, $\mathrm{n}(\%)$ & $19(27.1)$ & $49(70)$ \\
Mortality & & $16(22.9)$ \\
$\quad$ Yes, $\mathrm{n}(\%)$ & & $54(77.1)$ \\
$\quad$ No, $\mathrm{n}(\%)$ & & $35(50)$ \\
ESBLs colonization & & $35(50)$ \\
$\quad$ Positive, $\mathrm{n}(\%)$ & $18(25.7)$ & \\
$\quad$ Negative, $\mathrm{n}(\%)$ & $52(74.3)$ &
\end{tabular}

On discharged from ICU (Table 5), we found that ESBL colonization was associated to respiratory system dysfunction $(p=0.008, R R=1.987)$, third-generation cephalosporin usage $(p=0.009$, $R R=2.909)$, cefoperazone prescription $(p<0.001$, $R R=8.471)$, ceftriaxone prescription $(p=0.007$, $R R=6.316)$, and antibiotics usage duration $\geq 3$ days $(p<0.001, R R=7.071)$.

Table 3: Comparative analysis of ESBLs colonization on admission and discharge from ICU

\begin{tabular}{lllll}
\hline Variable & ESBL & & $\mathrm{p}$ & RR 95\% Cl \\
\cline { 2 - 3 } & Positive & Negative & & \\
\hline ESBL & & & & \\
$\begin{array}{c}\text { Admission } \\
\text { Discharge }\end{array}$ & $35(50)$ & $35(50)$ & 0.003 & $1.994(1.225-3.086)$ \\
\hline
\end{tabular}

ESBLs: Extended-spectrum beta-lactamases, ICU: Intensive care unit, RR: Risk ratio, Cl: Confidence interval.

The logistic regression results on influence of antibiotics usage and respiratory system dysfunction to ESBL colonization rate shows that both variables are independent risk factor to EBLS colonization both on admitted to and discharged from ICU (Table 6).

\section{Discussion}

In this study, we identified and compared the ESBL-producing Enterobacteriaceae colonization on patients who were admitted and discharged from the ICU. Positive ESBL-producing Enterobacteriaceae colonization was found on 18 patients (25.7\%) when 
admitted to ICU. The number was increased to 35 patients $(74.3 \%)$ on discharge. Thus, we found that antibiotics usage in critically ill patients was strongly associated to ESBL-producing Enterobacteriaceae colonization. The result was in line with a study by Harris et al. [8] which found 23 patients (23.7\%) from a total of 97 patients became ESBL-producing Enterobacteriaceae carriers during ICUs stay. Young et al. [9] also observed similar results on his study in Singapore and concluded that ICUs stay was the risk factor of ESBL-producing Enterobacteriaceae colonization.

Table 4: Variables of ESBL colonization on ICU admission

\begin{tabular}{|c|c|c|c|c|}
\hline \multirow[t]{2}{*}{ Variable $(n, \%)$} & \multicolumn{2}{|c|}{ ESBL on admission } & \multirow[t]{2}{*}{$p$ value } & \multirow[t]{2}{*}{$\mathrm{RR}(95 \% \mathrm{Cl})$} \\
\hline & Positive & Negative & & \\
\hline \multicolumn{5}{|l|}{ Sex } \\
\hline Male & $9(22.5)$ & $31(77.5)$ & \multirow[t]{2}{*}{0.477} & 0.750 \\
\hline Female & $9(30)$ & $21(70)$ & & $(0.339-1.658)$ \\
\hline \multicolumn{5}{|l|}{ Age } \\
\hline Adult & $14(24.6)$ & $43(75.4)$ & \multirow[t]{2}{*}{0.644} & 0.798 \\
\hline Geriatric & $4(30.8)$ & $9(69.2)$ & & $(0.314-2.031)$ \\
\hline \multicolumn{5}{|c|}{ Central nervous system dysfunction } \\
\hline Present & $10(22.2)$ & $35(77.8)$ & \multirow[t]{2}{*}{0.370} & 0.694 \\
\hline Absent & $8(32)$ & $17(68)$ & & $(0.315-1.531)$ \\
\hline \multicolumn{5}{|c|}{ Respiratory system dysfunction } \\
\hline Present & $12(41.4)$ & $17(58.6)$ & \multirow[t]{2}{*}{0.012} & 2.828 \\
\hline Absent & $6(14.6)$ & $35(85.4)$ & & $(1.200-6.661)$ \\
\hline \multicolumn{5}{|c|}{ Cardiovascular system dysfunction } \\
\hline Present & $9(26.5)$ & 25 (73.5) & \multirow[t]{2}{*}{0.888} & 1.059 \\
\hline Absent & $9(25)$ & $27(75)$ & & $(0.478-2.348)$ \\
\hline \multicolumn{5}{|c|}{ Gastrointestinal system dysfunction } \\
\hline Present & $7(38.9)$ & $11(61.1)$ & \multirow[t]{2}{*}{0.138} & 1.838 \\
\hline Absent & $11(21.2)$ & $41(78.8)$ & & $(0.841-4.016)$ \\
\hline \multicolumn{5}{|c|}{ Urogenital system dysfunction } \\
\hline Present & $5(31.3)$ & $11(68.7)$ & \multirow[t]{2}{*}{0.564} & 1.298 \\
\hline Absent & $13(24.1)$ & 41 (75.9) & & $(0.545-3.091)$ \\
\hline \multicolumn{5}{|c|}{ Musculoskeletal system dysfunction } \\
\hline Present & $3(16.7)$ & $15(83.3)$ & \multirow[t]{2}{*}{0.578} & 0.308 \\
\hline Absent & $15(28.8)$ & $37(71.2)$ & & $(0.189-1.767)$ \\
\hline \multicolumn{5}{|c|}{ Endocrine system dysfunction } \\
\hline Present & $5(50)$ & $5(50)$ & \multirow[t]{2}{*}{0.058} & 2.308 \\
\hline Absent & $13(21.7)$ & $47(78.3)$ & & $(1.053-5.057)$ \\
\hline Malignancy & & & & \\
\hline Present & $5(29.4)$ & $12(70.6)$ & 0.689 & 1.199 \\
\hline Absent & $13(24.5)$ & $40(75.5)$ & & $(0.500-2.876)$ \\
\hline Immune system dysfun & & & & \\
\hline Present & $0(0)$ & $5(100)$ & 0.172 & 1.383 \\
\hline Absent & $18(27.7)$ & $47(72.3)$ & & $(1.190-1.608)$ \\
\hline Corticosteroid usage & & & & \\
\hline Present & $4(33.3)$ & $8(66.7)$ & 0.507 & 1.381 \\
\hline Absent & $14(24.1)$ & $44(75.9)$ & & $(0.505-3.469)$ \\
\hline Antibiotics & & & & \\
\hline Cephalosporin & $16(30.8)$ & $36(69.2)$ & 0.100 & $2.769(0.705-$ \\
\hline Non-cephalosporin & $2(11.1)$ & $16(88.9)$ & & $10.885)$ \\
\hline Antibiotics prescription & mission & & & \\
\hline Ceftriaxone & $9(21.4)$ & $33(78.6)$ & 0.001 & \\
\hline Cefoperazone & $7(70)$ & $3(30)$ & & \\
\hline Cefazolin & $0(0)$ & $13(100)$ & & \\
\hline Others & $2(40)$ & $3(60)$ & & \\
\hline Antibiotics usage durat & & & & \\
\hline$\geq 3$ days & $10(37.0)$ & $17(63)$ & 0.086 & 1.991 \\
\hline$<3$ days & $8(18.6)$ & $35(81.4)$ & & $(0.899-4.410)$ \\
\hline
\end{tabular}

Some statistically significant correlations could be observed in our study as the risk factor of ESBL-producing Enterobacteriaceae colonization in ICU patients. The previous studies throughout the world also demonstrated the increment of ESBLproducing Enterobacteriaceae colonization associated to cephalosporin usage [9], [10], [11]. A study in Croatia showed that ceftriaxone use was significantly correlated with ESBL occurrence $(p<0.05)$ and concluded that ceftriaxone derestriction increased the occurrence of ESBLs and the utilization of carbapemens [12].

Antibiotic usage with duration $>3$ days increases the risk of ESBL-producing Enterobacteriaceae
Table 5: Variables of ESBL colonization on ICU discharge

\begin{tabular}{|c|c|c|c|c|}
\hline \multirow[t]{2}{*}{ Variable } & \multicolumn{2}{|c|}{ ESBL colonization } & \multirow[t]{2}{*}{$p$-value } & \multirow[t]{2}{*}{ RR $95 \% \mathrm{Cl}$} \\
\hline & Positive & Negative & & \\
\hline \multicolumn{5}{|l|}{ Sex } \\
\hline Male & $22(55)$ & $18(45)$ & \multirow[t]{2}{*}{0.334} & \multirow[t]{2}{*}{$1.269(0.773-2.084)$} \\
\hline Female & $13(43.3)$ & $17(56.7)$ & & \\
\hline \multicolumn{5}{|l|}{ Age } \\
\hline Adult & $28(49.1)$ & $29(50.9)$ & \multirow[t]{2}{*}{0.759} & \multirow[t]{2}{*}{$0.912(0.517-1.611)$} \\
\hline Geriatric & $7(53.8)$ & $6(46.2)$ & & \\
\hline \multicolumn{5}{|c|}{ Central nervous system dysfunction } \\
\hline Present & $25(53.1)$ & $18(41.9)$ & \multirow[t]{2}{*}{0.086} & \multirow[t]{2}{*}{$1.570(0.903-2.730)$} \\
\hline Absent & $10(37.0)$ & $17(63)$ & & \\
\hline Respiratory syste & & & & \\
\hline Present & $25(64.1)$ & $14(35.9)$ & 0.008 & $1.987(1.133-3.484)$ \\
\hline Absent & $10(32.3)$ & $21(67.7)$ & & \\
\hline Cardiovascular s & tion & & & \\
\hline Present & $17(54.8)$ & $14(45.2)$ & 0.470 & $1.188(0.746-1.893)$ \\
\hline Absent & $18(46.2)$ & $21(53.8)$ & & \\
\hline Gastrointestinal s & tion & & & \\
\hline Present & $10(52.6)$ & $9(47.4)$ & 0.788 & $1.074(0.645-1.788)$ \\
\hline Absent & $25(49.0)$ & $26(51)$ & & \\
\hline Urogenital systen & & & & \\
\hline Present & $9(52.9)$ & $8(47.1)$ & 0.780 & $1.079(0.638-1.825)$ \\
\hline Absent & $26(49.0)$ & $27(51)$ & & \\
\hline Musculoskeletal & ction & & & \\
\hline Present & $9(69.2)$ & $4(30.8)$ & 0.124 & $1.518(0.958-2.404)$ \\
\hline Absent & $26(89.7)$ & $3(10.3)$ & & \\
\hline Endocrine systen & & & & \\
\hline Present & $5(45.5)$ & $6(54.5)$ & 0.743 & $0.894(0.757-1.958)$ \\
\hline Absent & $30(50.8)$ & $29(49.2)$ & & \\
\hline Malignancy & & & & \\
\hline Present & $12(57.1)$ & $9(42.9)$ & 0.434 & $1.217(0.757-1.958)$ \\
\hline Absent & $23(46.9)$ & $26(53.1)$ & & \\
\hline Immune system & & & & \\
\hline Present & $2(66.7)$ & $1(33.3)$ & 0.555 & $1.354(0.587-3.124)$ \\
\hline Absent & $33(49.3)$ & $34(50.7)$ & & \\
\hline Corticosteroid us & & & & \\
\hline Present & $7(70)$ & $3(30)$ & 0.172 & $1.500(0.921-2.433)$ \\
\hline Absent & $28(46.7)$ & $32(53.3)$ & & \\
\hline Central venous $\mathrm{C}$ & & & & \\
\hline Present & $20(58.8)$ & $14(41.2)$ & 0.151 & $1.412(0.880-2.280)$ \\
\hline Absent & 15 (41.7) & $21(58.3)$ & & \\
\hline Endotracheal tub & & & & \\
\hline Present & $29(49.2)$ & $30(50.8)$ & 0.743 & $0.901(0.495-1.640)$ \\
\hline Absent & $6(54.5)$ & $5(45.6)$ & & \\
\hline Peripheral intrave & & & & \\
\hline Present & $31(47.7)$ & $34(52.7)$ & 0.164 & $0.596(0.359-0.990)$ \\
\hline Absent & $4(80)$ & $1(20)$ & & \\
\hline Nasogastric tube & & & & \\
\hline Present & $32(53.3)$ & $28(46.7)$ & 0.172 & $1.778(0.670-4.717)$ \\
\hline Absent & $3(30)$ & $7(70)$ & & \\
\hline Hemodialysis & & & & \\
\hline Present & $4(66.7)$ & $2(33.3)$ & 0.393 & $1.376(0.741-2.558)$ \\
\hline Absent & $31(48.4)$ & $33(51.6)$ & & \\
\hline Mechanical venti & & & & \\
\hline Present & $12(63.2)$ & $7(36.8)$ & 0.179 & $1.400(0.886-2.214)$ \\
\hline Absent & $23(45.1)$ & $28(54.9)$ & & \\
\hline Third-generation & usage & & & \\
\hline Yes & $32(58.2)$ & $23(41.8)$ & 0.009 & $2.909(1.032-8.203)$ \\
\hline No & $3(20)$ & $12(80)$ & & \\
\hline Antibiotics usage & & & & \\
\hline Ceftriaxone & $20(52.8)$ & $18(47.4)$ & 0.007 & $6.316(0.944-42.252)$ \\
\hline Cefoperazone & $12(70.6)$ & $5(29.4)$ & $<0.001$ & $8.471(1.265-56.715)$ \\
\hline Cefazolin & $1(8.3)$ & $11(91.7)$ & - & - \\
\hline Others & $2(66.7)$ & $1(33.3)$ & 0.024 & $8.000(1.040-61.525)$ \\
\hline Antibiotics usage & & & & \\
\hline$\geq 3$ days & $33(67.3)$ & $16(32.7)$ & $<0.001$ & $7.071(1.865-26.807)$ \\
\hline$<3$ days & $2(9.5)$ & $19(90.5)$ & & \\
\hline
\end{tabular}

colonization rate 7 -fold higher $(p<0.001, R R=7.071)$. Patients with respiratory system dysfunction are also at increasing risk to be carriers $(p=0.008, R R=1.987)$. It may be associated to the third-generation cephalosporin usage such as cefoperazone and ceftriaxone as empirical antibiotic to treat pneumonia. An observational

Table 6: Results of logistic regression on influence of antibiotics usage and respiratory system dysfunction to ESBL colonization rate

\begin{tabular}{llll}
\hline ESBL colonization & Variables tested & p-value & RR 95\% Cl \\
\hline ESBL on admission & Antibiotics usage & 0.062 & $4.895(0.926-25.885)$ \\
& Respiratory system dysfunction & 0.008 & $5.056(1.529-16.712)$ \\
ESBL on discharge & Antibiotics usage & 0.018 & $5.606(1.342-23.425)$ \\
& Respiratory system dysfunction & 0.013 & $3.773(1.322-10.769)$ \\
\hline ESBL: Extended-spectrum beta-lactamase, RR: Risk ratio, Cl: Confidence interval.
\end{tabular}


multicenter study in France showed similar result with a significant correlation between ESBL-producing Enterobacteriaceae colonization and respiratory system dysfunction $(p<0.01)$, urogenital system dysfunction ( $p<0.01)$, endocrine system dysfunction ( $p$ $<0.01$ ), and immune system dysfunction ( $p<0.01)$. Our study, however, reported significant correlation only on patients with respiratory system dysfunction [13].

In our study, invasive procedure variable analysis shows no significant correlation to ESBLproducing Enterobacteriaceae colonization with central venous catheter usage $(p=0.151)$, endotracheal intubation $(p=0.743)$, peripheral IV line $(p=0.164)$, nasogastric tube placement $(p=0.172)$, hemodialysis $(p=0.393)$, and mechanical ventilator $(p=0.179)$. The previous literatures showed various results in correlation with invasive procedure. Kawano et al. [14] and Repesse et al. [15] showed that mechanical ventilator $(p=0.476$ and $p=0.1$, respectively) had no statistically significant correlation to ESBL-producing Enterobacteriaceae colonization incidence. Another study, however, showed a different result that invasive procedure had strong correlation to ESBL-producing Enterobacteriaceae colonization with central venous catheters $(p<0.01)$, hemodialysis $(p<0.01)$, and mechanical ventilator ( $p$ $<0.01$ ). The different result may be caused by the brief utilization of the invasive tools [13], [14], [15]. Further studies with larger sample size would help demonstrate the relationship of invasive procedure and ESBLproducing Enterobacteriaceae colonization.

Some limitations in our study included the fact that we collected no environmental sample that could cause ESBL-producing Enterobacteriaceae colonization by direct contact. The study was carried out only in ICU patients and no subsequent observations of morbidity and mortality were done after the patients were discharged from ICU.

\section{Conclusion}

The antibiotics usage and respiratory system dysfunction are independent risk factors to EBLS colonization in ICU patients.

\section{References}

1. Mulki SS, Ramamurthy K, Bhat S. Fecal carriage of extendedspectrum beta-lactamase-producing Enterobacteriaceae in intensive care unit patients. Indian J Crit Care Med. 2017;21(8):525-7. https://doi.org/10.4103/ijccm.ijccm_112_17 PMid:28904483

2. Suranadi IW, Fatmawati NN, Aryabiantara IW, Sinardja CD,
Saputra DJ. Acinetobacter baumannii is an opportunistic pathogen as an MDRO in ICU. Bali $\mathrm{J}$ Anesthesiol. 2019;3(2):150-3. https://doi.org/10.15562/bjoa.v3i2.199

3. McDanel J, Schweizer M, Crabb V. Incidence of extendedspectrum $\beta$-lactamase (ESBL)-producing Escherichia coli and Klebsiella infections in the United States: A systematic literature review. Infect Control Hosp Epidemiol. 2017;38(10):1209-15. https://doi.org/10.1017/ice.2017.156

PMid:28758612

4. Fouda R, Soliman MS, EIAnany MG, Abadeer M, Soliman G. Prevalence and risk factors of MRSA, ESBL, and MDR bacterial colonization upon admission to an Egyptian medical ICU. J Infect Dev Ctries. 2016;10(4):329-36. https://doi.org/10.3855/ jidc.6798

PMid:27130993

5. Drinka P, Niederman MS, El-Solh AA, Crnich CJ. Assessment of risk factors for multi-drug resistant organisms to guide empiric antibiotic selection in long term care: A dilemma. J Am Med Dir Assoc. 2011;12(5):321-5. https://doi.org/10.1016/j. jamda.2010.06.012

PMid:21450192

6. Kalluru S, Eggers S, Barker A, Shirley D, Sethi AK, Sengupta S, et al. Risk factors for infection with multidrug-resistant organisms in Haryana, India. Am J Infect Control. 2018;46(3):341-5. https:// doi.org/10.1016/j.ajic.2017.08.021 PMid:29102426

7. Singh N, Pattnaik D, Neogi DK, Jena J, Malick B. Prevalence of ESBL in Escherichia coli isolates among ICU patients in a tertiary care hospital. J Clin Diagn Res. 2016;10(9):DC19-22. https://doi.org/10.7860/jcdr/2016/21260.8544

PMid:27790433

8. Harris AD, Kotetishvili M, Shurland S, Johnson JA, Morris JG Nemoy LL, et al. How important is patient-to-patient transmission in extended-spectrum $\beta$-lactamase Eschericia coli acquisition. Am J Infect Control. 2007;35(2):97-101. https://doi. org/10.1016/j.ajic.2006.09.011

PMid:17327188

9. Young BE, Lye DC, Krishnan P, Chan SP, Leo YS. A prospective observational study of the prevalence and risk factors for colonization by antibiotic resistant bacteria in patients at admission to hospital in Singapore. BMC Infect Dis. 2014;14(1):298. https://doi.org/10.1186/1471-2334-14-298 PMid:24889720

10. Hamprecht A, Rohde AM, Behnke M, Feihl S, Gastmeier P, Gebhardt $F$, et al. Colonization with third-generation cephalosporin-resistant Enterobactericeae on hospital admission: Prevalence an risk factors. J Antimicrob Chemother. 2016;71(10):2957-63. https://doi.org/10.1093/jac/dkw216 PMid:27317445

11. Bilavsky E, Temkin E, Lerman Y, Rabinovich A, Salomon J, Lawrence $\mathrm{C}$, et al. Risk factors for colonization with extendedspectrum beta-lactamase-producing Enterobacteriaceae on admission to rehabilitation centres. Clin Microbiol Infect. 2014;20(11):O804-10. https://doi.org/10.1111/1469-0691.12633 PMid:24674024

12. Skrlin J, Vrca VB, Marusic S, Ciric-Crncec MC, Mayer L. Impact of ceftriaxone de-restriction on the occurrence of ESBL-positive bacterial strains and antibiotic consumption. J Chemother. 2011;23(6):341-4. https://doi.org/10.1179/joc.2011.23.6.341 PMid:22233817

13. Barbier F, Pommier C, Essaied W, Garrouste-Orgeas M, Schwebel C, Ruckly S, et al. Colonization and infection with extended-spectrum $\beta$-lactamase-producing Enterobacteriaceae in ICU patients: What impact on outcomes and carbapenem exposure? J Antimicrob Chemother. 2016;71(4):1088-97. https://doi.org/10.1093/jac/dkv423 
PMid:26755492

14. Kawano $\mathrm{Y}$, Nishida T, Togawa A, Irie Y, Hoshino K, Matsumoto N, et al. Surveillance of extended-spectrum $\beta$-lactamaseproducing Enterobacteriaceae carriage in a Japanese intensive care unit: A retrospective analysis. Korean J Crit Care Med. 2016;31(4):317-23. https://doi.org/10.4266/kjccm.2016.00703
15. Repesse $X$, Artiguenave M, Paktoris-Papine S, Espinasse $F$, Dinh A, Charron C, et al. Epidemiology of extended-spectrum beta-lactamase-producing Enterobactericeae in an intensive care unit with no single rooms. Ann Intensive Care. 2017;7(1):73. https://doi.org/10.1186/s13613-017-0295-0

PMid:28674848 